\title{
Biologia e tabela de vida de fertilidade de Frankliniella occidentalis (Pergande) (Thysanoptera, Thripidae) em morangueiro
}

\author{
Aline Nondillo ${ }^{1}$, Luiza Rodrigues Redaelli ${ }^{1}$, Silvia Marisa Jesien Pinent ${ }^{1} \&$ Marcos Botton $^{2}$
}

${ }^{1}$ Programa de Pós-graduação em Fitotecnia, Departamento de Fitossanidade-UFRGS, Av. Bento Gonçalves 7.712, 91540-000 Porto Alegre-RS, Brasil. alinondillo@yahoo.com.br; luredael@ufrgs.br; silviapi@portoweb.com.br

${ }^{2}$ Embrapa Uva e Vinho, Rua Livramento 515, Caixa Postal 130, 95700-000 Bento Gonçalves-RS, Brasil. marcos@cnpuv.embrapa.br

\begin{abstract}
Biology and fertility life table of Frankliniella occidentalis (Pergande) (Thysanoptera, Thripidae) in strawberry. This work aimed to study biological aspects of Frankliniella occidentalis (Pergande) (Thysanoptera, Thripidae) considering that almost nothing is known about the thrips fauna associated with strawberry crop. Newly hatched larvae were individualized into Petri dishes, containing a flower or a foliole of strawberry and kept in chambers $\left(25 \pm 1{ }^{\circ} \mathrm{C}, 60 \pm 10 \% \mathrm{RH} ; 12\right.$ hours photophase). Daily observations were conducted until the insect death. The average time of the biological cycle (larva-adult) and viability did not differ between the insects maintained in flowers $(8.49 \pm$ 0.18 and $68.52 \%)$ and folioles $(8.85 \pm 0.15$ and $75.47 \%)$. The daily and total average fecundity were higher when flowers were provided as food $(7.4 \pm 0.69$ and $70.0 \pm 9.18 \mathrm{eggs} /$ femeale respectively $)$ compared with folioles $(2.4 \pm 0.35$ and 8.5 $\pm 1,13$ eggs/femeale respectively). The average time, in days, of the embryonic period was significantly different for the insects reared in flowers $(3.7 \pm 0.03)$ than in folioles $(4.4 \pm 0.09)$. The viability of eggs deposited in flowers and folioles was $65.5 \pm 0.01$ and $74.3 \pm 0.03 \%$ respectively. Based upon the fertility life table, the performance of F. occidentalis reared in flowers was better when compared to folioles. A generation (egg-adult) occurs each 20.92 days at $25{ }^{\circ} \mathrm{C}$.
\end{abstract}

KEYWORDS. Fragaria x ananassa; life of cycle; Thripidae; thrips.

\begin{abstract}
RESUMO. Biologia e tabela de vida de fertilidade de Frankliniella occidentalis (Pergande) (Thysanoptera, Thripidae) em morangueiro. O objetivo deste trabalho foi estudar aspectos biológicos de Frankliniella occidentalis (Pergande) (Thysanoptera, Thripidae), considerando que no Brasil quase nada se sabe sobre a fauna de tripes associada à cultura do morangueiro. Larvas recém-eclodidas foram individualizadas em placas de Petri contendo uma flor ou um folíolo de morangueiro, mantidas em câmaras climatizadas $\left(25 \pm 1{ }^{\circ} \mathrm{C} ; 60 \pm 10 \%\right.$ U.R.; fotofase de 12 horas $)$ e observadas diariamente até a morte. A duração média do período de larva-adulto e a viabilidade não diferiram entre os insetos mantidos em flores $(8,49 \pm 0,18$ e $68,52 \%)$ e folíolos $(8,85 \pm 0,15$ e $75,47 \%)$. A fecundidade média diária e a total foram mais elevadas quando flores foram fornecidas como alimento $(7,4 \pm 0,69$ e 70,0 \pm 9,18 ovos/fêmea respectivamente), em comparação com folíolos $(2,4 \pm 0,35$ e $8,5 \pm 1,13$ ovos/fêmea, respectivamente). A duração média, em dias, do período embrionário foi distinta entre os indivíduos mantidos em flores $(3,7 \pm 0,03)$ e em folíolos $(4,4 \pm 0,09)$. A viabilidade dos ovos depositados sobre flores e folíolos foi de $65,5 \pm 0,01$ e 74,3 $\pm 0,03 \%$, respectivamente. Com base na tabela de vida de fertilidade, o desempenho dos indivíduos de $F$. occidentalis que se desenvolveram em flores foi melhor, com uma geração (ovo-adulto) completada a cada 20,92 dias, a $25{ }^{\circ} \mathrm{C}$.
\end{abstract}

PALAVRAS-CHAVE. Ciclo de vida; Fragaria x ananassa; Thripidae; tripes.

O morangueiro (Fragaria x ananassa Duchesne) (Rosaceae) é uma planta típica de climas frios, com ampla distribuição geográfica, cujo cultivo tem se expandido nos últimos anos no Brasil (Calvete et al. 2005). A possibilidade de utilização dos frutos, tanto no setor industrial, como para consumo "in natura", aliada ao gosto atrativo e à riqueza de vitaminas e minerais, têm contribuído para o aumento do consumo e da produção, sendo considerada em escala mundial, a mais importante das chamadas pequenas frutas (Padovani 1991).

Uma das principais áreas de produção de morangos no Brasil está concentrada no estado do Rio Grande do Sul com aproximadamente 700 ha de área cultivada (Emater 2004). No estado, destacam-se os municípios do vale do rio Caí como produtores de morangos de mesa, seguidos por Caxias do Sul e Farroupilha, enquanto que em Pelotas e nos municípios vizinhos a produção é de morango-indústria (Pagot \& Hoffmann 2003).

Dentre os insetos que reduzem a qualidade dos frutos do morangueiro, destaca-se Frankliniella occidentalis (Pergande) (Thysanoptera: Thripidae), devido à alta freqüência com que ocorre, especialmente, em flores (Pinent et al. 2005). Originária da Califórnia (Estados Unidos da América) e do México, essa espécie atualmente encontra-se distribuída por vários países do mundo, tendo se estabelecido progressivamente no Sul do Brasil desde 1993 (Monteiro et al. 1999). Na região da Serra, no Rio Grande do Sul, foi registrada por Pinent et al. (2005) em caquizeiro, videira e morangueiro.

Frankliniella occidentalis é altamente polífaga e vetora do vírus do vira-cabeça do tomateiro (TSWV) (Moritz et al. 2004). Em morangueiro, os danos associados à espécie são descritos como decorrentes da alimentação, que podem causar, nas flores e frutos, bronzeamentos seguidos de murchamento (Gonzales-Zamora \& Garcia-Mari 2003). Ainda, em relação aos danos há opiniões contraditórias, principalmente, no que diz respeito ao senso comum de atribuir ao inseto deformações nos frutos (Coll et al. 2006). 
O controle de tripes nas áreas infestadas não tem sido tarefa fácil devido ao seu pequeno tamanho, rápido crescimento populacional e hábitos crípticos (Coll et al. 2006). O manejo da espécie no morangueiro vem sendo feito através da aplicação de inseticidas de alta toxicidade, muitas vezes não autorizados para uso na cultura. Esta situação, além de contaminar os frutos e o ambiente, onera o custo de produção e acarreta desequilíbrios ambientais, como a eliminação de polinizadores e inimigos naturais.

O conhecimento das características biológicas de uma espécie praga é fundamental para o desenvolvimento de estratégias eficientes de manejo e controle. Registros desta natureza, relativos a $F$. occidentalis foram obtidos para as culturas do amendoinzeiro, pepineiro, feijão-de-porco, repolho, pimenteiro e feijoeiro (Lowry et al.1992; Gaum et al. 1994; Lopes \& Alves 2000; Zhang et al. 2007). No entanto, não existem informações referentes à biologia da espécie associada à cultura do morangueiro, especialmente no Rio Grande do Sul, segundo maior produtor do país (Calvete et al. 2005). Neste trabalho foi estudada a biologia de $F$. occidentalis em flores e folíolos de morangueiro, calculando-se a tabela de vida de fertilidade.

\section{MATERIALE MÉTODOS}

O trabalho foi conduzido no Laboratório de Biologia, Ecologia e Controle Biológico de Insetos e na área experimental do Departamento de Fitossanidade da UFRGS, em Porto Alegre $\left(30^{\circ} 01^{\prime}\right.$ S, 51 $\left.1^{\circ} 13^{\prime} \mathrm{O}\right)$, Rio Grande do Sul. Na área, manteve-se uma cultura de morangueiro (Fragaria $X$ ananassa) cultivar Aromas, da qual eram retiradas as flores e folíolos utilizados tanto na criação como no estudo de biologia. No laboratório, foi estabelecida a criação de $F$. occidentalis e desenvolvido o experimento de biologia. A criação teve inicio com os insetos coletados em flores de morangueiros da cultivar Aromas, de Santa Lúcia do Piai (29 $10^{\circ}$ S, 51 $1^{\circ} 10^{\prime}$ O), Caxias do Sul, RS. Em laboratório, os insetos foram retirados do material vegetal vindo do campo e transferidos para placas de Petri $(4,4 \mathrm{~cm}$ de diâmetro X 2,5 cm de altura) com novas flores ou folíolos provenientes da área experimental, que serviram tanto de substrato de alimentação, quanto de oviposição. A cada dois dias, este material era substituído e mantido em outras placas até a eclosão das larvas.

Para determinação da duração e viabilidade das fases imaturas, larvas recém-eclodidas, provenientes da criação, foram individualizadas, com auxílio de um pincel, em placas de vidro (4,4 cm de diâmetro x $2,5 \mathrm{~cm}$ altura), contendo uma flor ou um disco de folíolo de morangueiro ( $3 \mathrm{~cm}$ de diâmetro), utilizados como substrato de alimentação. Estes substratos foram colocados sobre uma porção de papel filtro $\left(225 \mathrm{~mm}^{2}\right)$ umedecido com água destilada. As placas foram fechadas com parafilme e mantidas em câmara climatizada $\left(25 \pm 1{ }^{\circ} \mathrm{C} ; 60 \pm\right.$ $10 \%$ U.R.; fotofase de 12 horas). Acompanhou-se diariamente o desenvolvimento de 54 larvas em flores e $53 \mathrm{em}$ folíolos. O alimento foi substituído a cada três dias, ou antes, deste intervalo, quando perdia a turgidez. A separação dos sexos foi realizada observando-se as características morfológicas dos adultos recém-emergidos.

Para a obtenção dos dados referentes à fecundidade, fertilidade, duração dos períodos de pré-oviposição, oviposição, pós-oviposição e longevidade, adultos recémemergidos, provenientes de folíolos (27 fêmeas e 13 machos), e flores (29 fêmeas e 8 machos) foram individualizados e alimentados nas mesmas condições dos imaturos, e acompanhados até a morte.

Diariamente os adultos eram observados e os folíolos e flores substituídos. Os substratos de alimentação/oviposição retirados das placas das fêmeas eram acondicionados, individualmente, numa outra placa de vidro, com papel filtro $\left(225 \mathrm{~mm}^{2}\right)$ umedecido com água destilada, fechada com parafilme e identificada com a data e o número da fêmea.

Tendo em vista que as posturas são endofíticas, o registro da fecundidade e da fertilidade foi efetuado, num primeiro momento, computando-se diariamente o número de indivíduos eclodidos presentes em cada uma das placas que continham os folíolos. Quatro dias após a eclosão da última larva, o folíolo passava por um processo de clareamento, através da imersão por 2 horas em álcool $70 \%$. Posteriormente, a contagem total dos ovos ou córions remanescentes era realizada em estereomicroscópio, com o auxílio de um transiluminador. Os ovos depositados sobre as flores, observados apenas nas sépalas, foram contados sem a necessidade de clareamento utilizando-se o mesmo equipamento. Diariamente, as larvas eclodidas eram contadas e removidas das placas.

Os dados foram testados quanto à normalidade pelo teste Shapiro-Wilk, quando a distribuição da variável apresentou normalidade foi utilizado o teste $\mathrm{t}$ de Student e em caso contrário o teste de Mann-Whitney. A razão sexual (rs) foi calculada através da fórmula, $\mathrm{rs}=\mathrm{n}^{\circ}$ de fêmeas $/ \mathrm{n}^{\circ}$ de fêmeas $+\mathrm{n}^{\mathrm{o}}$ de machos, e, a tabela de vida de fertilidade, conforme Silveira Neto et al. (1976). Para estas análises foram utilizados os softwares Microsoft ${ }^{\circledR}$ Excel 2000, Bioestat ${ }^{\circledR} 4.0$ e SPSS $^{\circledR}$ for Windows 15.0.

Os exemplares de F. occidentalis foram identificados com base em Mound \& Marullo (1996) e as lâminas de microscopia encontram-se depositadas, como testemunhas, na coleção do referido laboratório na UFRGS.

\section{RESULTADOSEDISCUSSÃO}

A duração do primeiro instar larval foi significativamente menor $(\mathrm{t}=4,585, \mathrm{P}=0,0001)$ nos insetos alimentados em flores de morangueiro (Tabela I). Sobre folíolos, o valor médio (2,0 \pm 0,06 ) foi superior aos $1,1 \pm 0,3$ dias obtidos por Lowry et al. (1992), em amendoinzeiro, a $25^{\circ} \mathrm{C}$. No segundo instar, o tempo de desenvolvimento não diferiu entre os indivíduos mantidos nos dois órgãos avaliados ( $\mathrm{t}=0,732, \mathrm{P}=0,466)$ (Tabela I). Lowry et al. (1992) observaram duração média similar (3,6 1, 1,0 dias).

Os diferentes órgãos vegetais oferecidos não afetaram a duração dos estágios de pré-pupa $(\mathrm{t}=1,699, \mathrm{P}=0,093)$ e pupa $(t=0,192, P=0,842)$ (Tabela I) como era esperado, pois de acordo com Lewis (1973) nestas fases os insetos não se 
Tabela I. Duração média em dias ( \pm EP), intervalo de variação (IV) e viabilidade média (\% \pm EP), das fases de larva, pré-pupa, pupa e ciclo biológico (larva-adulto) de Frankliniella occidentalis mantidos em flores e folíolos de morangueiro da cultivar Aromas, ( $\mathrm{n}=$ número de observações) (25 $\pm 1{ }^{\circ} \mathrm{C} ; 60 \pm 10 \%$ U.R.; fotofase 12 horas).

\begin{tabular}{|c|c|c|c|c|c|c|}
\hline \multirow[t]{2}{*}{ Estágio } & \multicolumn{3}{|c|}{ Flor } & \multicolumn{3}{|c|}{ Folíolo } \\
\hline & Duração (n) & IV & Viabilidade & Duração (n) & IV & Viabilidade \\
\hline $1^{\circ}$ instar & $1,6 \pm 0,07$ a $(54)$ & $1-3$ & $96,3 \pm 2,62 \mathrm{a}$ & $2,0 \pm 0,06 \mathrm{~b}(53)$ & $1-3$ & $96,2 \pm 2,64 \mathrm{a}$ \\
\hline $2^{\circ}$ instar & $3,3 \pm 0,15$ a $(52)$ & $1-6$ & $86,5 \pm 4,78 a$ & $3,5 \pm 0,20$ a (51) & $1-10$ & $88,2 \pm 4,56 \mathrm{a}$ \\
\hline Pré-pupa & $1,1 \pm 0,05$ a (45) & $1-2$ & $91,1 \pm 4,29 a$ & $1,0 \pm 0,02$ a (45) & $1-3$ & $95,6 \pm 3,11 \mathrm{a}$ \\
\hline Pupa & $2,4 \pm 0,11 \mathrm{a}(41)$ & $1-4$ & $90,2 \pm 4,69 \mathrm{a}$ & $2,4 \pm 0,09$ a $(43)$ & $1-4$ & $93,0 \pm 3,93 \mathrm{a}$ \\
\hline Ciclo Biológico & $8,49 \pm 0,18$ a (37) & $7-11$ & $68,52 \pm 2,89 a$ & $8,85 \pm 0,15$ a $(40)$ & $7-11$ & $75,47 \pm 1,41 \mathrm{a}$ \\
\hline
\end{tabular}

${ }^{1}$ Médias seguidas de mesma letra na linha não diferem significativamente pelo teste $\mathrm{t}$ de Student, a $5 \%$ de probabilidade

alimentam nem excretam. O ciclo de vida nos tisanópteros é intermediário entre os insetos holometábolos e hemimetábolos, sendo ainda notáveis por apresentarem mais de um estágio pupal (Mound \& Marullo 1996). Provavelmente e por estes motivos, os valores registrados para o morangueiro se aproximam aos relatados por Lowry et al. (1992) e Gaum et al. (1994), para as fases de pré-pupa (1,0 $\pm 0,0$ e 1,04 $\pm 0,028$ dias $)$ e pupa $(2,0 \pm 0,6$ e 2,96 $\pm 0,028$ dias $)$ em amendoinzeiro e pepineiro, respectivamente.

A viabilidade média de ambos os instares larvais e das fases de pré-pupa e pupa não diferiram significativamente entre os indivíduos mantidos em flores e folíolos de morangueiro (Tabela I).

A duração média do ciclo biológico (larva-adulto) e a viabilidade foram semelhantes, entre os insetos mantidos em flores e folíolos (P>0,05) (Tabela I). Lowry et al. (1992) e Gaum et al. (1994) observaram para F. occidentalis, valores de duração do ciclo biológico superiores ao do presente estudo, entretanto, próximos entre eles, quando os indivíduos foram criados apenas sobre folhas de amendoinzeiro $(13,8 \pm 0,6$ dias $)$ e de pepineiro (14,7 $\pm 0,13$ dias), respectivamente. Zhi et al. (2005) também não encontraram diferença significativa na sobrevivência de imaturos de F. occidentalis quando vagens de feijoeiro e pólen de macieira foram oferecidas isoladamente ou em conjunto.

Embora o efeito dos diferentes recursos alimentares oferecidos não tenha sido significativo na duração do ciclo biológico (larva-adulto) suas implicações na biologia são marcantes. $\mathrm{O}$ alimento ingerido na fase larval afeta, não só o tempo de desenvolvimento, mas também o tamanho corporal do tripes. Assim, larvas maiores e mais rápidas são potencialmente menos suscetíveis a serem predadas por seus inimigos naturais (Gerin et al. 1999; de Kogel et al. 1999; Hulshof et al. 2003).

Os diferentes órgãos vegetais avaliados, não afetaram significativamente a longevidade de machos $(\mathrm{t}=1,816, \mathrm{P}=$ $0,85)$ e fêmeas $(\mathrm{t}=1,023, \mathrm{P}=0,311)$ (Tabela II). Machos e fêmeas mantidos em folíolos tiveram longevidade semelhante $(\mathrm{t}=0,537, \mathrm{P}=0,600)$, porém, as fêmeas mantidas em flores foram significativamente $(\mathrm{t}=3,315, \mathrm{P}=0,003)$, mais longevas do que os machos.

Trichilo \& Leigh (1988) e Hulshof et al. (2003) demonstraram que a adição de pólen no alimento de $F$. occidentalis resultou em maior longevidade. Entretanto, Zhi et al. (2005) não detectaram essa influência. No presente estudo, as fêmeas foram mais longevas quando alimentadas nas flores, o que pode ser atribuído a presença de pólen.

Os insetos mantidos em flores e folíolos de morangueiro apresentaram uma razão sexual de 0,78 e 0,67 respectivamente, não diferindo significativamente $\left(\chi^{2}=1,147, \mathrm{P}=0,284\right)$.

O período de pré-oviposição foi similar $(\mathrm{U}=261,500, \mathrm{P}=$ $0,92)$ entre os insetos mantidos sobre os dois órgãos vegetais, entretanto, o de oviposição foi significativamente maior $(\mathrm{t}=$ $3,443, \mathrm{P}=0,001$ ) para fêmeas alimentadas com flores (Tabela II). O período médio de pós-oviposição também diferiu significativamente $(\mathrm{t}=1,569, \mathrm{P}=0,123)$ entre os dois grupos, sendo maior nos insetos mantidos sobre os folíolos (Tabela II).

$\mathrm{O}$ melhor desempenho reprodutivo de $F$. occidentalis ocorreu quando flores foram fornecidas como alimento, sendo significativamente mais elevadas a fecundidade média diária $(\mathrm{t}=6,367, \mathrm{P}=0,0001)$ e a total $(\mathrm{t}=6,652, \mathrm{P}=0,0001)$, quando comparadas com a registrada em folíolos (Tabela II).

Insetos tendem, de modo geral, a ovipositar sobre plantas hospedeiras que são nutricionalmente benéficas para o desenvolvimento de sua prole (Thompson 1988). Isto pode explicar as altas taxas de fecundidade observadas no presente trabalho, em flores. Reforçando esta idéia, Kirk (1996) relatou que em ambientes naturais, tisanópteros têm por costume ovipositar próximo às flores, apesar destas não serem um recurso estável na natureza e, normalmente, estarem restritas a curtos espaços de tempo. Esse autor considera que a principal compensação às dificuldades de viver em flores é a possibilidade de obtenção de um recurso alimentar de melhor qualidade. Segundo Bernays \& Chapman (1994), a qualidade do alimento encontrado nas flores é superior ao de outras partes vegetais, devido à intensa transferência de nitrogênio dos órgãos vegetativos para os reprodutivos. Grãos de pólen têm altas concentrações de nitrogênio e a preferência por este alimento é freqüentemente citada como uma das principais formas para obtenção de uma dieta rica em proteínas, podendo promover um aumento na produção de ovos, na taxa de crescimento e na longevidade de muitas espécies de tripes (Kirk 1984, 1996).

Esta hipótese foi corroborada pelos resultados obtidos por Trichilo \& Leigh (1988) e Hulshof et al. (2003), os quais verificaram um aumento significativo na fecundidade de $F$. occidentalis quando o pólen foi adicionado à dieta. Em $F$. shultzei, Milne et al. (1996) observaram o mesmo efeito. 
Tabela II. Longevidade de machos e fêmeas, períodos de pré-oviposição, oviposição, pós-oviposição (dias) ( \pm EP), fecundidade média diária e total, fertilidade média e respectivos intervalos de variação (IV), de Frankliniella occidentalis mantidos em flores e folíolos de morangueiro cultivar Aromas, $\left(\mathrm{n}=\right.$ número de observações) $\left(25 \pm 1{ }^{\circ} \mathrm{C} ; 60 \pm 10 \%\right.$; fotofase 12 horas $)$.

\begin{tabular}{|c|c|c|c|c|}
\hline \multirow[t]{2}{*}{ Parâmetro Biológico } & \multicolumn{2}{|c|}{ Flor } & \multicolumn{2}{|c|}{$\begin{array}{r}\text { Folíolo } \\
\end{array}$} \\
\hline & Média \pm EP (n) & IV & Média \pm EP $(n)$ & IV \\
\hline \multicolumn{5}{|l|}{ Longevidade(dias) } \\
\hline Fêmeas & $14,8 \pm 1,62 \mathrm{a} \mathrm{A}^{1}(29)$ & $1-31$ & $12,8 \pm 0,93$ a $\mathrm{A}(27)$ & $1-24$ \\
\hline Machos & $7,1 \pm 1,64$ a B $(8)$ & $4-18$ & $11,8 \pm 1,73$ a $\mathrm{A}(13)$ & $2-25$ \\
\hline \multicolumn{5}{|l|}{ Período(dias) } \\
\hline Pré-oviposiçãa & $2,5 \pm 0,16$ a $(26)$ & $1-4$ & $3,3 \pm 0,31$ a $(27)$ & $1-8$ \\
\hline Oviposição & $10,5 \pm 1,28 \mathrm{a}(26)$ & $1-23$ & $5,1 \pm 0,86 \mathrm{~b}(27)$ & $1-17$ \\
\hline Pós-oviposição & $3,3 \pm 0,62$ a $(26)$ & $1-11$ & $4,5 \pm 0,46 \mathrm{~b}(27)$ & $1-11$ \\
\hline \multicolumn{5}{|l|}{ Fecundidade(número) } \\
\hline Diária & $7,4 \pm 0,69$ a $(26)$ & $0,7-15$ & $2,4 \pm 0,35 \mathrm{~b}(27)$ & $0,3-8$ \\
\hline Total & $70,0 \pm 9,18$ a $(26)$ & $3-159$ & $8,5 \pm 1,13$ b (27) & $1-23$ \\
\hline Fertilidade $(\%)$ & $65,5 \pm 0,01$ a $(1821)$ & $1-8$ & $74,3 \pm 0,03 \mathrm{~b}(230)$ & $2-8$ \\
\hline
\end{tabular}

${ }^{1}$ Médias seguidas de mesma letra, minúsculas na linha e maiúsculas na coluna, não diferem significativamente pelo teste t de Student, a $5 \%$ de probabilidade.

Embora F. occidentalis, como muitos outros "tripes das flores", tenha o pólen como seu principal recurso alimentar (Grinfeld 1959; Kirk 1984), as altas taxas de fecundidade em flores também podem ser decorrentes da combinação de recursos oferecidos (sépalas, pétalas, estames, carpelos e pólen), uma vez que foram observadas marcas de alimentação deixadas pelos tripes em todas estas estruturas.

Os resultados de Milne et al. (1996) para Frankliniella shultzei (Trybom) (Thysanoptera: Thripidae) também corroboram esta hipótese, os autores constataram que as fêmeas alimentadas com pétalas de malvavisco eram tão fecundas quanto às alimentadas com pólen e, em uma dieta combinada de pólen, pétalas e folhas, a fecundidade foi significativamente maior, quando comparada àquelas contendo apenas um destes recursos.

Outros fatores que poderiam explicar o sucesso reprodutivo de $F$. occidentalis sobre flores é o fato de apresentarem tigmotactismo positivo e polifagia (Lewis 1973; Mound 2005). Assim, além do fornecimento de um alimento de melhor qualidade, as flores podem atuar como abrigo para estes insetos, oferecendo locais protegidos da ação de fatores meteorológicos e inimigos naturais.

Com relação à duração média do desenvolvimento embrionário, valores significativamente menores foram observados na prole dos indivíduos alimentados com flores de morangueiro $(3,7 \pm 0,03$ dias $)(t=7,383, P=0,0001)$ do que na dos mantidos em folíolos $(4,4 \pm 0,09$ dias). A diferença observada pode ser atribuída à nutrição das fêmeas, uma vez

Tabela III. Duração média de uma geração (T) taxa líquida de reprodução $\left(\mathrm{R}_{0}\right)$, razão infinitesimal de aumento (rm) e razão finita de aumento $(\lambda)$ para indivíduos de Frankliniella occidentalis mantidos em flores e folíolos de morangueiro cultivar Aromas $\left(25 \pm 1{ }^{\circ} \mathrm{C} ; 70 \pm 10 \%\right.$; fotofase 12 horas).

\begin{tabular}{lllll}
\hline ÓrgãoVegetal & $\mathrm{T}$ (dias) & $\mathrm{R}_{\mathrm{o}}$ & $\mathrm{r} \mathrm{m}$ & $\lambda$ \\
\hline Flor & 20,92 & 23,89 & 0,15 & 1,16 \\
Folíolo & 19,15 & 3,15 & 0,06 & 1,06 \\
\hline
\end{tabular}

que estas foram alimentadas desde a eclosão com o mesmo órgão da planta. Resultados similares foram obtidos por Hulshof et al. (2003), a $25^{\circ} \mathrm{C}$, quando comparou fêmeas de $F$. occidentalis alimentadas com pólen de três espécies de betuláceas, com as que receberam apenas folhas de pepineiro.

A viabilidade dos ovos de F. occidentalis foi significativamente maior nos ovos depositados nos folíolos, quando comparados aos depositados em flores $(\mathrm{F}=7,1726, \mathrm{P}$ $=0,0075)$ (Tabela II). A diferença pode ser atribuída à turgidez diferencial destes dois tipos de tecidos, já que a umidade é um fator fundamental que afeta a viabilidade de ovos (Parra 2007), sendo estes, de uma forma geral, mais suscetível ao ressecamento, que os demais estágios de desenvolvimento.

A duração média de uma geração de F. occidentalis variou em função do alimento oferecido, sendo maior em flores que em folíolos de morangueiro (Tabela III). A taxa líquida de reprodução $\left(\mathrm{R}_{0}\right)$ em folíolos, foi cerca de oito vezes menor do que em flores (Tabela III). A razão infinitesimal de aumento populacional $(\mathrm{rm})$ e a razão finita de aumento $(\lambda)$ foram maiores para os insetos mantidos em flores (Tabela III). Os resultados indicam que o desempenho geral dos indivíduos de $F$. occidentalis que se desenvolveram em flores de morangueiro foi melhor quando comparado ao dos mantidos em folíolos. Os valores são comparáveis aos obtidos por Trichilo \& Leigh (1988) e Hulshof et al. (2003) que constataram melhor desempenho reprodutivo de $F$. occidentalis, quando pólen foi adicionado sobre folhas de algodoeiro e pepineiro, respectivamente, a $25^{\circ} \mathrm{C}$.

$\mathrm{O}$ sucesso reprodutivo de $F$. occidentalis constatado em flores de morangueiro sugere que estas são, em campo, um alimento adequado ao desenvolvimento do inseto. Assim, não havendo condições limitantes de temperatura, a presença de flores na cultura do morangueiro potencializaria o crescimento populacional de F. occidentalis.

Agradecimentos. Os autores agradecem ao Conselho Nacional de Desenvolvimento Científico e Tecnológico (CNPq) pelo suporte financeiro (Projeto $\mathrm{n}^{\circ} 473433 / 2006-0$ ) e pela concessão de bolsas aos autores. À granja Andreazza e ao Sr. José Pazza pelo fornecimento de material biológico para a condução do trabalho. 


\section{REFERÊNCIAS}

Bernays, E. A. \& R. F. Chapman. 1994. Host-plant selection by phytophagous insects. New York, Chapman \& Hall, 312 p.

Calvete, E. O.; H. C. Rocha; O. T. Antunes \& A. A Nienow. 2005. Morangueiro polinizado pela abelha jataí em ambiente protegido. Passo Fundo, UPF, 53 p.

Coll, M.; S. Shakya; I. Shouster \& Y. Nenner. 2006. Decision-making tools for Frankliniella occidentalis management in strawberry: consideration of target markets. Entomologia Experimentalis et Applicata 121: 1-9.

de Kogel, W. J.; M. Mollemba \& C. van der Hoek. 1999. Effect of host plant on body size of Frankliniella occidentalis (Thysanoptera: Thripidae) and its correlation with reproductive capacity. European Journal of Entomolology 96: 365-368.

Emater. Levantamento da fruticultura comercial do Rio Grande do Sul - 2003/2004. 2004. Porto Alegre, Ascar Emater-RS, 89 p.

Gaum, W. G.; J. H. Giliomee \& K. L. Prngle. 1994. Life history and life table of western flower thrips, Frankliniella occidentalis (Thysanoptera: Thripidae), on English cucumbers. Bulletin of Entomological Research 84: 219-224.

Gerin, C.; T. H. Hance \& G. van Impe. 1999. Impact of flowers on the demography of western flower thrips Frankliniella occidentalis (Thy., Thripidae). Journal of Applied Entomology 123: 569_ 574.

Gonzales-Zamora, J. E. \& F. Garcia-Mari. 2003. The efficiency of several sampling methods for Frankliniella occidentalis (Thysanoptera: Thripidae) in strawberry flowers. Journal of Applied Entomology 127: 516-521.

Grinfeld, E. K. 1959. Feeding of thrips on the pollen of flowers and the origin of asymmetry in their mouthparts. Entomological Review 38: $715-720$.

Hulshof, J.; E. Ketoja \& Vännine. 2003. Life history chacteristics of Frankliniella occidentalis on cucumber leaves and without supplemental food. Entomologia Experimentalis et Applicata 108: $19-32$.

Kirk, W. D. J. 1984. Pollen-feeding in thrips (Insecta: Thysanoptera). Journal of Zoology 204: 107-117.

Kirk, W. D. J. 1996. Thrips. Naturalists' Handbooks. 1996. Slough, The Richmond Publishing Co.Ltd., 70 p.

Lewis, T. 1973. Thrips: their biology, ecology, and economic importance. London, Academic Press, 349 p.

Lopes, R. B. \& S. B. Alves. 2000. Criação e observações preliminares da biologia de Frankliniella occidentalis (Pergande) (Thysanoptera: Thripidae) em feijão-de-porco Canavalia ensiformis (L.). Anais da Sociedade Entomológica do Brasil 1: 39-47.

Lowry, V. K.; J. R. Smith Jr. \& F. L. Mitchele. 1992. Life-fertility tables for Frankliniella fusca (Hisnds) and Frankliniella occidentalis (Pergande) (Thysanoptera: Thripidae) on peanut. Annals of the Entomological Society of America 85: 744-754.

Milne, J. R.; G. H. Walter; D. J. Kaonga \& G. C. Sabio. 1996. The importance of non-pollen plant parts as food sources for the common blossom thrips, Frankliniella schultzei. Entomologia Experimentalis et Applicata 78: 271-281.

Monteiro, R. C.; L. A. Mound \& R. A. Zucchi. 1999. Thrips (Thysanoptera) as pests of plants production in Brazil. Revista Brasileira de Entomologia 43: 163-171.

Mound, L. A. 2005. Thysanoptera: diversity and interactions. Annual Review Entomology 50: 247-269.

Mound, L. A. \& R. Marullo 1996, The thrips of Central and South America: an introduction (Insecta: Thysanoptera). (Memoirs on Entomology International), $6^{\circ}$ vol., Associated Publishers, 487 p.

Moritz, G.; S. Kumm \& L. A. Mound. 2004. Tospovirus transmission depends on thrips ontogeny. Virus Research 100: 143-149.

Padovani, M. I. 1991. Morango: o delicado e saboroso fruto da integração dos povos. São Paulo, Ícone, 35 p.

Pagot, E. \& A. Hoffmann. 2003. Produção de pequenas frutas no Brasil. In: Seminário Brasileiro sobre Pequenas Frutas. Anais. Bento Gonçalves: Embrapa Uva e Vinho (Documentos 37). 64 p.

Parra, J. R. P. 2007. Técnicas de criação de insetos para programas de controle biológico. Piracicaba, FEALQ, 137 p.

Pinent, S. M. J.; M. Botton \& L. R. Redaelli. 2005. Thrips species (Thysanoptera) on Strawberry, Persimmon and Grape in the Gaúcho Highlands, Rio Grande do Sul State, Southern Brazil. In: Ullman D.; J. Moyer; R. Goldbach; G. Moritz. Journal of Insect Science 7: 28 .

Silveira Neto, S.; O. Nakano; D. Bardin \& N. A. Villa Nova. 1976. Manual de ecologia dos insetos. Piracicaba, Agronômica Ceres, 419 p.

Thompson, J. N. 1988. Evolutionary ecology of the relationship between oviposition preference and performance of offspring in phytophagous insects. Entomologia Experimentalis et Applicata 47: 3-14.

Trichillo, P. J. \& T. F. Leigh 1988. Influence of resource quality on the reproductive fitness of flower thrips (Thysanoptera: Thripidae). Annals of the Entomological Society of America 81: 64-71.

Zhang, Z. J.; Q. J. Wu; X. F. Li; Y. J. Zhang; B. Y. Xu \& G. R. Zhu. 2007. Life history of western flower thrips, Frakliniella occidentalis (Thy.:Thripidae), on five different vegetable leaves. Journal of Applied Entomology 131: 347-345.

Zhi, J.; G. K. Fitch; D. C Margolies \& J. R. Nechols. 2005. Apple pollen as a supplemental food for the western flower thrips, Frankliniella occidentalis: response of individuals and populations. Entomologia Experimentalis et Applicata 117: 185-192. 